\title{
Precision Medicine in BCR-ABL1 Negative Myeloproliferative Neoplasms
}

\author{
Linet Njue $^{1}$, Naomi Porret ${ }^{1}$, Ulrike Bacher ${ }^{1}$, Anne Angelillo-Scherrer ${ }^{1}$, Alicia Rovó ${ }^{1}$
}

\section{ABSTRACT}

Polycythemia vera, essential thrombocythemia, and primary myelofibrosis constitute BCR-ABL1-negative myeloproliferative neoplasms (MPNs) and are characterized by activation of the physiologic signal-transduction pathways resulting in cell proliferation of one or more of the hematopoietic lineages. The evolution of molecular studies has enlightened us in the understanding of this complex disease and has contributed to revisions of the World Health Organization (WHO) diagnostic criteria and risk stratification. A challenging aspect of these disorders is the diverse clinical features that occur in each disease type, with variability in risks of disease complications such as thrombosis and progression to fibrosis and/or leukemia.

The selection of the precise management for each patient is mandatory, and therefore, an accurate definition of diagnosis and prognostication is necessary. The clinical presentation, peripheral blood and chemical blood changes, the bone marrow histological features as well as the molecular/genomic profile are all aspects that contribute to a more precise way to diagnose and treat these patients.

This review attempts to summarize all those aspects that can contribute to the most detailed diagnosis and therapy approach in patients with classic BCR-ABL1 negative MPNs, emphasizing the relevance of interpretation of each step in the concept of personalized medicine.

Keywords: Polycythemia vera (PV), essential thrombocythemia (ET), primary myelofibrosis (PMF), myeloproliferative neoplasms (MPNs)

\section{INTRODUCTION}

Healthcare systems have focused for the last 10 years on addressing patient care, leaving the concept of a one-drug-fits-all model by identifying aspects inherent to patients and their diseases that might allow diagnosis and management optimization by offering customized care to each case. This healthcare model called precision medicine or personalized medicine is considered as particularly relevant for those diseases in which a marker can have a prognostic meaning or can represent a target for specific treatment. ${ }^{1}$ Hence, this model represents a current modern approach in the management of malignant diseases in general and thanks to the enormous contribution of molecular and genomic in hematological diseases in particular. ${ }^{2}$

Myeloproliferative neoplasms (MPNs) are clonal hematopoietic stem cell disorders characterized by activation of the physiologic signal-transduction pathways responsible for cell proliferation of one or more of the hematopoietic lineages. The diverse clinical features that occur in each MPN entity with variability in risks of disease complications and progression to secondary leukemia are challenging for physicians taking care of these patients. In MPNs, consideration of individual variability is imperative in diagnostics and management of the disease. The clinical presentation, the peripheral blood values and clinical chemistry parameters, the bone marrow's histological features as well as the molecular/ genomic profile are all aspects that might contribute to a more precise way to diagnose and treat these patients. ${ }^{3}$ This review attempts to summarize all those aspects that can contribute to the most detailed diagnosis and therapy approach in patients with classic BCR-ABL1 negative MPNs, emphasizing the relevance of interpretation of each step in the concept of personalized medicine.
${ }^{1}$ Department of Hematology and Central Hematology Laboratory, Inselspital, Bern University Hospital, University of Bern, 3010 Bern, Switzerland.

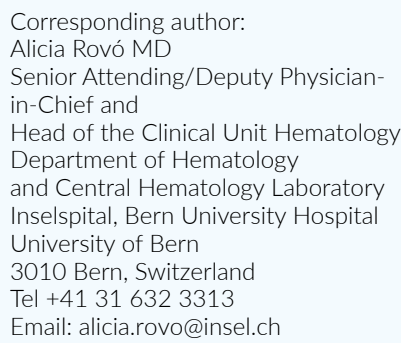

DOI:10.36000/hbT.OH.2020.06.024 ISSN: 2673-2092 (Print) and 26732106 (Online)

This article was published on 7th December, 2020

Njue $L$ et al. Precision Medicine in BCR-ABL1 Negative Myeloproliferative Neoplasms. healthbook TIMES Onco Hema 2020;(6):24-34 


\section{BLOOD COUNTS IN MPN}

In MPNs, a comprehensive evaluation of the peripheral blood counts reveals characteristic changes that can guide the diagnostic process from the very beginning. The presence of polyglobulia alone or accompanied by leukocytosis and thrombocytosis suggests the existence of an underlying polycythemia vera (PV). Isolated thrombocytosis is the classic presentation of essential thrombocythemia (ET) or a prefibrotic form of myelofibrosis (MF). MF in its initial form can present any type of cytosis, advanced forms in opposite are characterized by cytopenias. None of these peripheral blood changes are, however, specific for a distinct MPN. At presentation, cytosis in the context of an MPN should be differentiated from cytosis occurring due to reactive causes, which are significantly more frequent. In cases of presentation with cytopenia, the characteristic changes in the peripheral blood smears may help to guide towards the correct diagnosis, in these cases the main differential diagnosis is myelodysplastic syndrome (MDS).

The red blood cells (RBC) on their own can be informative. Michiels et al. could prove that an $\mathrm{RBC}$ count within the normal range $\left(<5.8 \times 10^{12} / \mathrm{L}\right.$ in males and $<5.6 \times 10^{12} / \mathrm{L}$ in females) may distinguish JAK2V617F positive ET from prodromal PV and overt PV. ${ }^{4}$

It has been demonstrated that changes in red cell distribution width (RDW) in patients with PV tend to be increased than in the normal controls when RBC were high. ${ }^{5}$ The presence of microcytosis in patients with high hemoglobin (Hb) levels could also be suggestive of PV associated with iron deficiency. Virtually all PV patients might have iron deficiency during the course of the disease. Iron metabolism in PV may be altered due to aberrant erythropoiesis, an inflammatory milieu, decreased systemic iron concentration, and potentially altered hypoxia responsiveness directly influencing iron absorption in the small intestine. ${ }^{6}$

Sandes et al. retrospectively analyzed 248,839 outpatients without known hematological disorders ${ }^{7}$ and were able to prove that the isolated use of the World Health Organization (WHO) proposed $\mathrm{Hb}$ / hematocrit (Hct) levels as a definer of polycythemia may lead to a substantial increase in unnecessary diagnostic tests. ${ }^{8}$ In cases with borderline levels of $\mathrm{Hb}$, the diagnostic workup of PV should only be initiated in the presence of clinical and/or laboratory features associated with MPNs.

In MPNs, the evaluation of blood smears may reveal leukocytosis, eosinophilia, basophilia, or the presence of immature myeloid forms. Likewise, quantitative or qualitative abnormalities of platelets are frequently observed, showing anisocytosis with large platelets and platelet degranulation. A leucoerythroblastic picture (i.e., teardrop-shaped RBC, circulating nucleated RBCs and/or immature myeloid cells) while not being pathognomonic, may suggest an advanced form of myelofibrosis.

\section{SYMPTOMS IN MPN PATIENTS}

The spectrum of MPNs-related symptoms is wide. These include constitutional symptoms (fever, night sweats, and weight loss), symptoms related to spleen enlargement (abdominal discomfort or pain and early satiety), symptoms related to microvascular disturbances (vertigo, lightheadedness, dizziness, insomnia, sexual dysfunction, numbness, tingling, headaches, and concentration problems), as well as fatigue, cough, bone pain, inactivity, and pruritus. Symptom heterogeneity may exist within each MPN subtype, sometimes independent of disease features or prognosis. ${ }^{9}$

Symptomatology of the patients can be assessed through the MPN Symptom Assessment Form (MPN-SAF or MPN10) questionnaire, a 10-item survey/symptom score, which has proved effective in capturing the presence and intensity of MPN-related symptoms. ${ }^{10}$ Each symptom is rated on a potential score of $0 /$ absent to 10 /worst imaginable. These ten core items include worst fatigue, early satiety, abdominal discomfort, concentration problems, inactivity, night sweats, itching, bone pain, fever, and weight loss. Although these symptoms are not taken into consideration in the WHO classification, using the MPN-10 score at each visit is an excellent way of objectifying the clinical follow-up in patients with MPNs. Thus, the presence of symptoms interfering with patient's quality of life should suggest the need to initiate or modify treatment.

Disease-related PV symptoms, such as microvascular disturbance, pruritus (which may be excruciating), migrainetype headache, and fatigue, can significantly impact the quality of life. ${ }^{11}$ However, patients may as well be asymptomatic at presentation. Constitutional symptoms in early PV are unusual, except for fatigue, which may have many causalities, including iron deficiency, thyroid or cardiovascular disease, sleep apnea, depression and pulmonary hypertension, and side effects related to the therapy used to treat the disease. ${ }^{12}$ Geyer et al. showed that patients with problematic PV (specifically hydroxyurea treatment failure, splenomegaly, or a persisting need for phlebotomies) have worse symptom burden compared with patients without these features. ${ }^{13}$

Most patients with ET are asymptomatic at diagnosis, and detection of thrombocytosis is typically incidental. Others present with disease-related vasomotor symptoms affecting microcirculation (e.g., headache, dizziness, visual changes, acral paresthesia) or thromboembolic or bleeding complications (e.g., bleeding first-trimester fetal loss). Unlike in PV, pruritus is very uncommon in ET, occurring in less than $5 \%$ of patients. Although ET has the lowest symptom severity, the prevalence of constitutional symptoms reported by patients is relatively high. ${ }^{12}$ Patients should be advised to modify their lifestyle where necessary, including stopping smoking, and should be recommended weight control and treatment of dyslipidemia and arterial hypertension if necessary. MF patients most frequently complain of severe fatigue, symptoms due to an 


\section{healthbook TIMES Oncology Hematology}

enlarged spleen, as well as weight loss, low-grade fever, bone pain, and night sweats. ${ }^{14,15} \mathrm{Up}$ to $30 \%$ of patients are initially asymptomatic. As the disease evolves, all patients become symptomatic due to marrow failure, increasing splenomegaly, and constitutional symptoms. In the advanced phases of MF, extramedullary hematopoiesis in sites other than the spleen and liver may be seen.

\section{MPN ENTITIES}

According to the WHO, the combination of clinical, morphological, and molecular genetic features is the most suitable attempt to define MPNs, with the importance of bone marrow morphological features being highlighted. Bone marrow morphology remains the central distinguishing feature in the 2016 WHO classification of MPNs. ${ }^{8}$

\section{Polycythemia vera}

$\mathrm{PV}$ is characterized by a molecular signature; all patients harbor the $J A K 2$ mutation, exceptionally in exon 12. Although other MPN entities can as well carry a $J A K 2$ mutation, the characteristic high allele burden can contribute to guide the diagnosis towards PV already at the time of first detection. Recently, another mutation was identified in $J A K 2$, which is characterized by a common 4-amino-acid deletion and variable 1-amino-acid insertion (Leu583-Ala586DelInsSer/Gln/ Pro) within the $J H 2$ domain. This novel recurrent mutation, albeit rare, was described in patients with eosinophilia, which fulfilled diagnostic criteria of both PV and chronic eosinophilic leukemia (CEL). ${ }^{16}$

The main changes in the revised 2016 WHO criteria are the $\mathrm{Hb} / \mathrm{Hct}$ threshold, the upgrade of bone marrow (BM) biopsy to a major criterion together with the presence of $J A K 2 \mathrm{~V} 617 \mathrm{~F}$ exon 14 , or $J A K 2$ exon 12 mutations. The subnormal serum erythropoietin level remains as a solely minor criterion since endogenous erythroid colony assay is no longer considered as a minor criterion. ${ }^{1}$ In the new classification, special importance is given to the histological evaluation of the BM in all MPNs, granting it a major criterion attribute for all MPN subtypes, including PV. In PV, the findings on BM examination change as the disease evolves from a prodromal pre-polycythemia phase with borderline to mild erythrocytosis to an overt polycythemic phase with increased red cell mass to a "spent" or post-polycythemic myelofibrosis with cytopenias, ineffective erythropoiesis, fibrosis, extramedullary hematopoiesis, and hypersplenism. ${ }^{17}$

\section{Masked PV \\ MPN patients may present with clinical features suggestive of PV such as abdominal thrombosis (Budd Chiari syndrome), isolated thrombocytosis, leukocytosis, or splenomegaly, but with $\mathrm{Hb}$ levels that do not reach the threshold defined by WHO. The lack of clear evidence of polyglobulia, may guide the diagnosis towards other MPNs than PV.}

Masked PV (mPV) was initially described as inapparent PV or latent PV..$^{18}$ Lamy $\mathrm{T}$ et al. observed that in certain situations, $\mathrm{Hb}$ and Hct levels remain normal even though there is a genuine case of polycythemia. A study conducted by Barbui et al. describes $\mathrm{mPV}$ as a possible variant of $\mathrm{PV}$ with lower $\mathrm{Hb}$ levels at baseline but with a trend to more frequent thrombotic events as well as more rapid progression to myelofibrosis and acute leukemia. ${ }^{19}$ Distinguishing ET from masked PV based on $\mathrm{Hb}$ or Hct values alone is rather impossible, yet this distinction is of utmost importance. Alvarez-Larran et al. showed that $\mathrm{mPV}$ could be identified using red blood cell mass (RCM) measurement. ${ }^{20}$ Cassinat et al. showed that a proportion of patients harboring JAK2 mutation had in fact early-stage polycythemia when RCM was systematically measured, these patients were erroneously classified as ET. ${ }^{21}$ The distinction between PV versus ET is indeed of clinical relevance with therapeutic impact since these patients should be managed as PV and not as ET, giving priority to Hct control, keeping it below $45 \%$ at least. This data underscores that in the era of molecular classification, the clinic continues to play a relevant role in the classification of MPNs. ${ }^{18}$ Other studies contributed to confirm this data, showing that once RCM is estimated, masked PV can turn out to be rather "unmasked" and a true $\mathrm{PV}^{22}$

$\mathrm{PV}$ may also present normal $\mathrm{Hb}$ values in some special situations, e.g., portal hypertension secondary to suprahepatic or portal vein thrombosis may present an expanded plasma volume that masks an increased RCM. ${ }^{23}$

\section{Essential thrombocythemia}

ET is characterized by excessive, clonal platelet production with a tendency to thrombosis and hemorrhage. Distinguishing reactive thrombocytosis from thrombocytosis associated with an underlying MPN is a critical step in patient's care. Since thrombocytosis may be the only abnormal finding, molecular testing should be performed for JAK2, CALR, and MPL. Especially in triple-negative cases, BM evaluation is necessary to identify the correct diagnosis. In ET, BM cellularity, size, and morphology of megakaryocytes contribute significantly to the diagnosis (Table 1). Patients with reactive thrombocytosis show neither spontaneous megakaryocyte nor erythroid colony growth. ${ }^{13}$

\section{Essential thrombocythemia versus prefibrotic/early primary myelofibrosis}

The distinction between prefibrotic myelofibrosis (prePMF) and ET may be difficult since both entities depict considerable overlap. The tendency of megakaryocytes to form clusters, as well as BM reticulin or collagen fibrosis, are both characteristics of pre-PMF but not ET helping, therefore in this differentiation (Table 1). Discrimination of ET from the prePMF still rests largely on proper histologic BM evaluation, ${ }^{24}$ as being highlighted in the revised WHO classification. ${ }^{8}$ Discrimination of both entities is clinically relevant because 
Table 1. Peripheral blood values, bone marrow characteristics, and chemical parameters in distinct MPNs. BM, bone marrow; ET, essential thrombocythemia; $\mathrm{Hb}$, hemoglobin; Hct, hematocrit; PMF, primary myelofibrosis; PV, polyycythemia vera; WBC, white blood count.

\begin{tabular}{|c|c|c|c|c|c|c|c|c|c|c|}
\hline & $\mathrm{Hb}$ & Hct & $\begin{array}{l}\text { Platelet } \\
\text { count }\end{array}$ & WBC & Blasts & LDH & Spleen & $\begin{array}{l}\text { BM biopsy } \\
\text { cellularity }\end{array}$ & $\begin{array}{l}\text { BM biopsy } \\
\text { fibrosis }\end{array}$ & $\begin{array}{l}\text { BM } \\
\text { megakaryocyte } \\
\text { morphology }\end{array}$ \\
\hline PV & $\begin{array}{l}>16.5 \mathrm{~g} / \mathrm{dL} \\
\text { in men } \\
>16.0 \mathrm{~g} / \mathrm{dL} \\
\text { in women }\end{array}$ & $\begin{array}{l}>49 \% \text { men } \\
>48 \% \\
\text { women }\end{array}$ & $\begin{array}{l}\text { possibly } \\
\text { raised }\end{array}$ & $\begin{array}{l}\text { possibly } \\
\text { raised }\end{array}$ & none & normal & $\begin{array}{l}\text { possibly } \\
\text { enlarged } \\
(70 \%)\end{array}$ & increased & $\begin{array}{l}\text { normal or } \\
\text { mild }\end{array}$ & $\begin{array}{l}\text { increased, } \\
\text { pleomorphic, loose } \\
\text { clusters }\end{array}$ \\
\hline ET & normal & normal & $\begin{array}{l}\geq 450 x \\
10^{\circ} / L\end{array}$ & normal & none & normal & $\begin{array}{l}\text { possibly } \\
\text { mildly } \\
\text { enlarged } \\
(50 \%)\end{array}$ & normal & $\begin{array}{l}\text { very rarely } \\
\text { grade } 1\end{array}$ & $\begin{array}{l}\text { increased, } \\
\text { enlarged (giant), } \\
\text { hyperlobulated } \\
\text { (staghorn-like) }\end{array}$ \\
\hline $\begin{array}{l}\text { pre- } \\
\text { PMF }\end{array}$ & $\begin{array}{l}\text { normal or } \\
\text { decreased }\end{array}$ & normal & $\begin{array}{l}\text { possibly } \\
\text { raised }\end{array}$ & $\begin{array}{l}\geq 11 X \\
10^{9} / L\end{array}$ & $\begin{array}{l}\text { not } \\
\text { increased }\end{array}$ & $\begin{array}{l}\text { discretely } \\
\text { raised }\end{array}$ & $\begin{array}{l}\text { possibly } \\
\text { enlarged }\end{array}$ & increased & $\begin{array}{l}\text { frequent } \\
\text { grade } 1\end{array}$ & $\begin{array}{l}\text { increased, } \\
\text { mostly enlarged, } \\
\text { loose clusters, } \\
\text { hypolobulation, } \\
\text { maturation defects, } \\
\text { naked nuclei }\end{array}$ \\
\hline PMF & decreased & $\begin{array}{l}\text { normal or } \\
\text { decreased }\end{array}$ & $\begin{array}{l}\text { both } \\
\text { raised and } \\
\text { decreased }\end{array}$ & $\begin{array}{l}\geq 11 x \\
10^{9} / L\end{array}$ & $\begin{array}{l}\text { possibly } \\
\text { raised }\end{array}$ & raised & $\begin{array}{l}\text { enlarged } \\
>19 \mathrm{~cm}(9)\end{array}$ & $\begin{array}{l}\text { normal, } \\
\text { decreased } \\
\text { or } \\
\text { increased } \\
(10-19 \%)\end{array}$ & $\begin{array}{l}\text { grade } \\
2-3\end{array}$ & $\begin{array}{l}\text { normal or increased } \\
\text { dense clusters, } \\
\text { mostly enlarged, } \\
\text { hypolobulation, } \\
\text { maturation defects, } \\
\text { naked nuclei }\end{array}$ \\
\hline
\end{tabular}

pre-PMF has a much higher probability of progressing to myelofibrosis and transforming into secondary acute leukemia compared to ET. ${ }^{25,26}$ Additionally, a JAK2V617F allele burden $>50 \%$ may favor a diagnosis of pre-PMF. ${ }^{24}$

\section{Prefibrotic/early primary myelofibrosis versus primary myelofibrosis (overt fibrotic stage)}

The distinction of pre-PMF from overt PMF due to their different survival and risk of disease progression has clinical relevance. ${ }^{27}$ Guglielmelli et al. analyzed data of 661 PMF patients and clearly showed differences between both entities. Patients with pre-PMF are younger compared with those with overt-PMF. Patients with overt-PMF have greater hematologic abnormalities (anemia, leukopenia, thrombocytopenia, higher blast counts), more frequently show clinical symptoms, have larger spleens, and have a higher frequency of molecular highrisk profiles. ${ }^{27} \mathrm{~W}$ ith this information, the clinician will be able to categorize patients more correctly and therefore optimize treatment decisions. This further augments the importance of $\mathrm{BM}$ evaluation in all patients newly diagnosed with MPNs.

\section{BIOCHEMISTRY}

In ET, signs of hypermetabolism with increased lactate dehydrogenase $(\mathrm{LDH})$ and uric acid levels are infrequent ${ }^{28,29}$ while they are typical in PMF. ${ }^{30}$ Mazzotta et al. found that the mean of LDH level was moderately increased in patients with PV and ET and markedly increased in PMF when compared with secondary polycythemia. ${ }^{31}$ This is important since increased LDH in PV and ET is associated with increased bone marrow proliferation and increased risk of progression to myelofibrosis and is also an independent predictor of survival. ${ }^{32,33}$ Considerable elevation of serum LDH independently predicts shorter overall and leukemiafree survival in PMF as well. ${ }^{34}$

Chronic inflammation has been associated with MPNs. ${ }^{35}$ In a study of 244 patients with PV and ET, the major thrombosis rate was significantly increased in patients with increased acute-phase protein high sensitivity C-reactive protein (CRP) levels. The highest levels of this biomarker doubled the risk of thrombosis. Conversely, high pentraxin 3 (PTX-3) levels are associated with lower rates of thrombosis. ${ }^{36}$ Patients with high CRP levels exhibit an increased risk of progressive disease and 3-4-fold increased mortality rate. ${ }^{37}$ These easily measurable biomarkers might, therefore, be useful to improve the prognostic classification of MPN patients.

In $3-14 \%$ of MPN patients, a monoclonal gammopathy can be identified ${ }^{38-41}$, probably coincidentally ${ }^{39}$ though one study has reported its presence to be associate with an adverse MPN disease course. ${ }^{41}$

\section{BONE MARROW FOLLOW-UP}

Bone marrow morphology remains the cornerstone of MPN diagnosis, ${ }^{8}$ but is also important during the follow-up under therapy. Successful interferon therapy for myelofibrosis has been associated with a significant reduction of marrow fibrosis, cellularity, megakaryocyte density, and naked nuclei density. ${ }^{42}$ Similarly, ruxolitinib therapy may reduce the amount of bone marrow fibrosis in a large proportion of patients with MPNassociated MF or may delay its progression.

\section{MPN-ASSOCIATED COMPLICATIONS}

Bleeding

Patients with any type of MPN can develop bleeding complications, ${ }^{43}$ and bleeding can even be the initial presentation of MPN, conducting to the MPN diagnosis. ${ }^{43,44}$ The prevalence of bleeding among patients newly diagnosed with MPN varies considerably across studies. ${ }^{45-49} \mathrm{~A}$ recent review and meta-analysis involving a total of 13,436 patients with MPN reported that a pooled prevalence of bleeding at a diagnosis of $6.2 \% .^{50}$ Minor or major hemorrhage events may also occur during the course of the disease. ${ }^{43}$ 


\section{healthbook TIMES Oncology Hematology}

The underlying pathophysiological involved mechanisms are frequently multifactorial. Since MPNs mainly affects elderly patients, comorbidities and polypharmacy may contribute to bleeding complications.

Within the disease-specific risk factors for bleeding, thrombocytosis is a leading risk factor. ${ }^{51}$ Bleeding risk may be worsened by the development of an acquired von Willebrand syndrome (aVWS), which may result from increased proteolysis with platelet activation leading to reduced von Willebrand factor activity. ${ }^{52}$ Some studies suggest consideration of aVWS even in the absence of extreme thrombocytosis. ${ }^{53}$ Thus, investigation of aVWS should be considered before administration of aspirin. CALR mutation has been associated with higher levels of thrombocytosis. However, in a retrospective study, the mutational status (CALR vs $J A K 2 \mathrm{~V} 617 \mathrm{~F})$ did not predict bleeding complications. ${ }^{51}$ Leukocytosis has been identified as bleeding risk factor mainly in patients with ET and early PMF. ${ }^{54}$

Treatments used in MPN patients can also trigger bleeding complications. Data evaluating the risk of bleeding of antithrombotic treatment is, however, controversial. Vitamin $\mathrm{K}$ antagonists (VKA) with or without aspirin did not increase the risk of bleeding in two large studies, ${ }^{49,55}$ whereas another study demonstrated a trend towards a higher rate of major bleeding in MPN patients treated with VKA compared to those not anticoagulated. ${ }^{56}$

The use of low dose aspirin to treat MPNs may be associated with increased bleeding complications. However, low-dose aspirin did not affect the risk of bleeding in PV patients in an European multicenter trial. ${ }^{57}$

Bleeding related to cytoreductive treatments, either hydroxyurea, interferon, or ruxolitinib, might be due to thrombocytopenia while bleeding under anagrelide therapy is more likely related to altered platelet function. ${ }^{58}$

In order to prevent bleeding in MPN patients, it might be wise to conduct aVWS investigation before prescribing low dose aspirin when the platelet count is above $1000 \times 10^{9} / \mathrm{L}$ and to consider testing for aVWS even with lower thrombocytosis. A cytoreduction to lower the platelet count can be administered in the presence of aVWS. Supportive therapies such as desmopressin and von Willebrand factor concentrate are usually given to patients with aVWS and active bleeding. In addition, platelet transfusion might be considered in bleeding complications if an underlying platelet dysfunction is suspected. ${ }^{52}$

The data summarized here reflects the need for an individualized assessment of the bleeding/thrombosis risk in each patient and emphasizes the need for tailored therapeutic decisions.

\section{Thrombotic complications}

Patients with MPNs have increased risks of thrombotic complications compared to age- and sex-matched individuals without MPNs. The occurrence of thrombosis is one of the main contributors to the increased morbidity and mortality in MPN patients. Thrombosis can be one of the initial manifestations that lead to the diagnosis of MPN. ${ }^{44,59}$ The reported prevalence of thrombosis among patients newly diagnosed with MPN varies considerably across studies. ${ }^{45-49}$ A meta-analysis, including 29 cohort studies with 13,436 MPN patients, showed that the pool prevalence of thrombosis was around $20 \%{ }^{50}$ The mechanisms associated with the occurrence of thrombosis in MPN patients are considered to be multifactorial; indeed, many aspects remain insufficiently clear and biological studies frequently show contradictory data. Uncontrolled cytosis, cell proliferation and cell activation of any hematopoietic lineage, exacerbation of underlying inflammation; changes in the coagulation system, abnormal cell adhesion, as well as participation of vascular endothelial factors are postulated to be participating factors. ${ }^{60}$ Importantly, whereas erythrocytosis ${ }^{61}$ and leukocytosis ${ }^{62-65}$ have been associated with thrombosis in several studies, thrombocytosis appears not as strongly correlated with the thrombotic risk. ${ }^{63}$ However, the increased thromboxane synthesis observed in PV platelets ${ }^{66}$ suggests that thromboxane-dependent platelet activation contributes to the increased risk of thrombosis among PV patients.

A retrospective study of 1,537 Chinese MPN patients showed that presence of a $J A K 2 \mathrm{~V} 617 \mathrm{~F}$ allele burden above $50 \%$ was significantly more frequent in patients with thrombosis compared to those without thrombotic complications. ${ }^{67}$ Thrombosis in patients with MPN could present as mild microcirculatory disturbance or as major arterial and venous thromboembolic events such as ischemic stroke, myocardial infarction, peripheral arterial diseases, and venous thromboembolism. ${ }^{68}$ MPN patients are at not only a high risk of developing arterial and venous thromboembolism but may develop as well thromboses at unusual sites such as the hepatic, portal, and splenic veins, the cerebral sinuses, and the mesenteric arteries. Patients with splanchnic vein thrombosis or cerebral sinus vein thrombosis may lack myeloproliferation but carry already MPN driving mutations at diagnosis. $J A K 2 \mathrm{~V} 617 \mathrm{~F}$ mutation is the driving mutation, which is the most frequently associated with unusual site thrombosis. ${ }^{69-73}$ Testing for $J A K 2 \mathrm{~V} 6127 \mathrm{~F}$ mutation is therefore usually performed in patients with splanchnic vein thrombosis, and testing for CALR may be restricted to JAK2V617F-negative patients with a spleen size of at least $16 \mathrm{~cm}$ and a platelet count above $200 \times 10^{9} /$ L. $^{74}$

Patients may be assigned to a thrombotic risk category depending on the patient- and disease-related variables. ${ }^{75}$ Accordingly, the thrombotic risk has an important weight on therapeutic decisions in patients with PV and ET. On the contrary, in MF, the thrombotic risk is not consistently included in the therapeutic algorithm. In PV, age and history of previous thrombosis are the prognostic factors used to classify patients in low-risk and high-risk categories. In ET, 

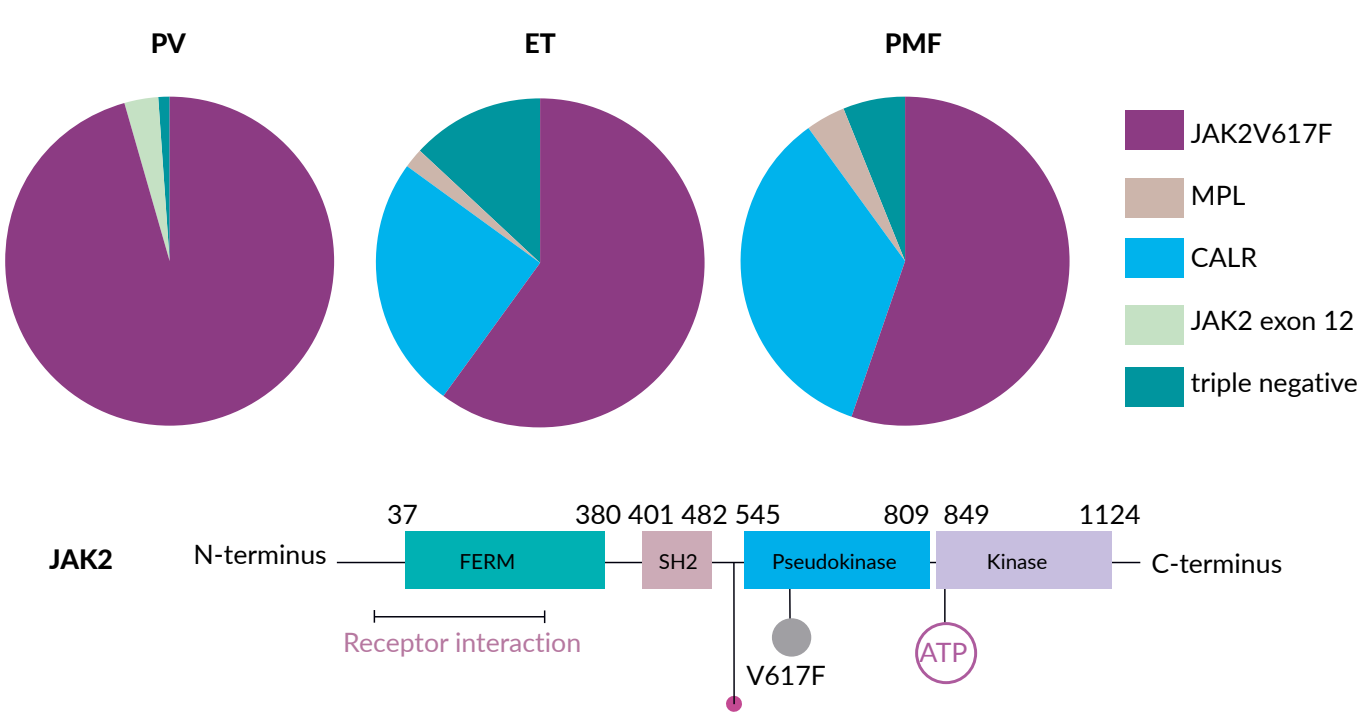

Exon 12 mutation
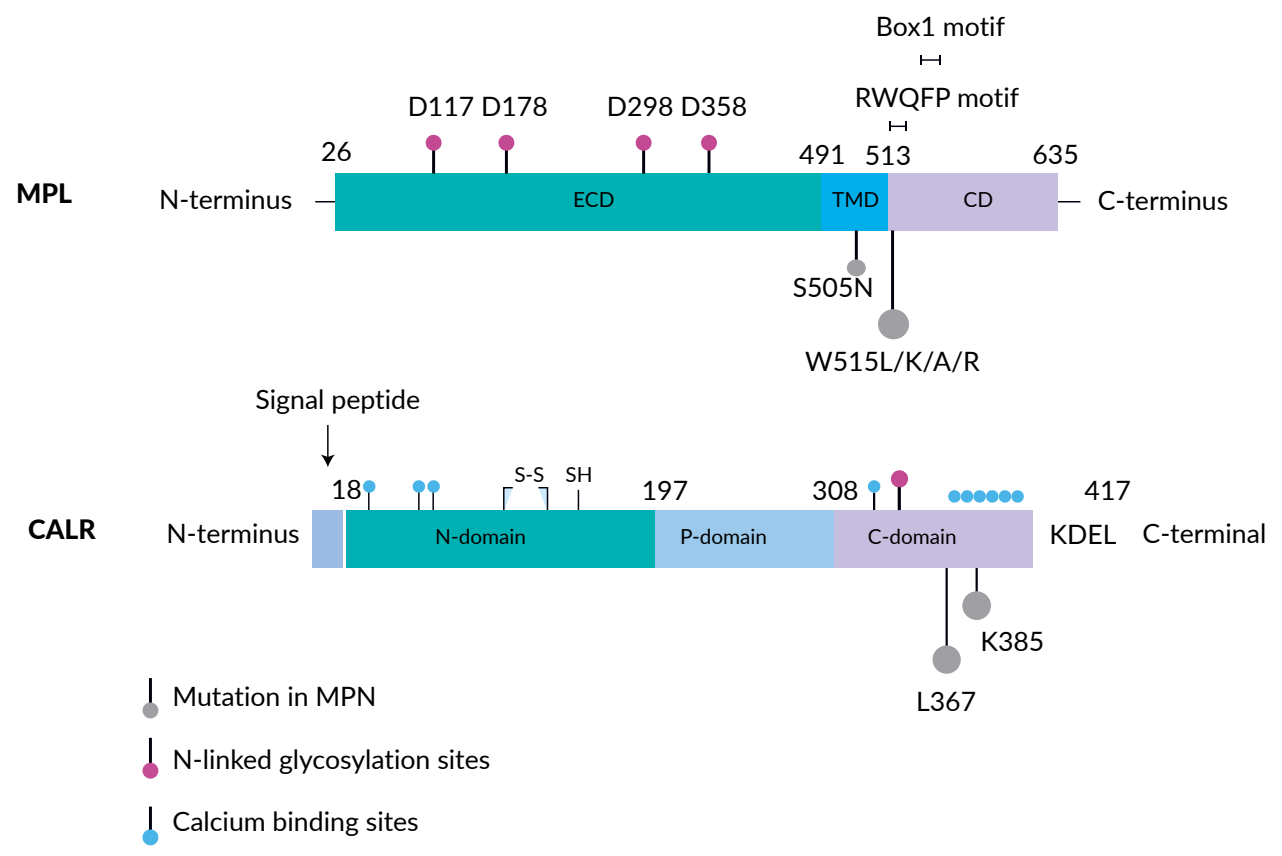

Figure 1. Disease driver mutations in the classical MPNs. JAK2, MPL and CALR mutations account for the vast majority of the classical MPN cases. (with permission from Ruochen Jia \& Robert Kralovics). 11

the international prognosis score of thrombosis (IPSET) system is the recommended prognostic system comprising age, previous thrombosis, cardiovascular risk factors, and JAK2V617F mutation. ${ }^{76-79}$ It should be applied to all ET patients at diagnosis. Furthermore, control of general risk factors for thrombosis, including smoking habits, diabetes mellitus, arterial hypertension, and hypercholesterolemia, is as well recommended. ${ }^{80}$

\section{MYELOPROLIFERATIVE NEOPLASMS DISEASE TRANSFORMATION}

MPNs can evolve into fibrotic forms or have a clonal evolution transforming into more serious myeloid diseases such as acute myeloid leukemia (AML). ${ }^{81-84}$ Some MPNs like ET show a more benign course and transform less frequently; however, fibrotic post-ET has been described. Previously, many ET patients had no BM histological evaluation at time of diagnosis. Thus, we may hypothesize that some of these patients were incorrectly labeled as ET despite having, probably, a prefibrotic form of MF instead. The most recent WHO updates ${ }^{8}$ suggest systematic BM trephine biopsy investigation at first presentation. This will allow a better estimation of the real risk of fibrotic transformation of ET in the future. Long-lasting PV may progress to a spent phase, called post PV-myelofibrosis; clear risk factors for this progression are not well defined. Myelofibrosis as an entity per se, may initially present in an early form, with little evidence of fibrosis, and progress over time into an overt form. 


\section{healthbook TIMES Oncology Hematology}

MPNs can progress to secondary AML. The recognition of potential risk factors for such a dramatic transformation is of great interest. It is being considered that this risk is influenced by factors related to the disease and the treatments received as well. A nationwide MPN cohort study evaluating 11,039 patients in Sweden showed that the risk of AML/MDS development after MPN diagnosis was significantly associated with high exposures of Phosphorus-32 $\left({ }^{32} \mathrm{P}\right)$ and alkylators but not with hydroxyurea $(\mathrm{HU})$ treatment. Likewise, this study showed that $25 \%$ of MPNs patients who developed AML/ MDS were not exposed to cytotoxic therapy, supporting a significant role for factors without relation to therapy. ${ }^{85}$ Postulated disease-related factors include mutations of $C A L R$, splicing factors, epigenetics, and signaling molecules of RAS proteins. ${ }^{86-88} \mathrm{~A}$ recent study evaluating the longterm outcome of 3,023 consecutive MPN patients showed a leukemic transformation in $6 \%$. The incidence of leukemic transformation was significantly higher in patients with PMF compared to those with PV $(9.3 \%$ vs $3.9 \% ; P<0.001)$ and ET (9.3\% vs $2.6 \% ; P<0.001)$ but remained comparable in patients with PV versus those with ET $(P=0.22) .{ }^{84}$

Table 2. IPSET score: International prognostic score for thrombosis in essential thrombocytopenia (ET) ${ }^{76}$

\begin{tabular}{|c|c|c|}
\hline \multicolumn{3}{|l|}{ 1. Calculate IPSET-thrombosis score } \\
\hline Patient age? & under 60 years & 60 years or older \\
\hline Prior thrombotic event? & No & Yes \\
\hline Cardiovascular risk factors present & No & Yes \\
\hline JAK2V617F mutation detected? & No & Yes \\
\hline
\end{tabular}

One or more of: hypertension, diabetes mellitus, active smoking

\begin{tabular}{|l|l|l|}
\hline \multicolumn{2}{|l|}{ IPSET-thrombosis score: } \\
\hline \multicolumn{2}{|l|}{ 2. Determine risk goup and prognosis } \\
\hline Score & Risk category & Annual thrombosis risk \\
\hline $0-1$ & Low & $1.03 \%$ \\
\hline 2 & Intermediate & $2.35 \%$ \\
\hline $3-6$ & High & $3.56 \%$ \\
\hline
\end{tabular}

\section{MOLECULAR INVESTIGATIONS IN MPNS}

Role of driver mutations for precision medicine

Already in 1960, the Philadelphia chromosome was discovered by Hungerford and Nowell, discriminating CML from the other, $B C R-A B L 1$ negative MPNs being discussed in this chapter. ${ }^{89}$ However, more than four decades passed by before the JAK2V617F was detected in PV. ${ }^{90,91}$ This was followed in 2006 by the identification of the next genetic variant: MPLW515L and W515K. ${ }^{22}$ The next molecular discovery followed in 2013 by two separate research groups: CALR mutations in exon 9, leading to a frameshift resulting in a novel C-terminal peptide sequence. ${ }^{15,93}$ All three mutations $J A K 2$, $M P L$ and $C A L R$, affect the JAK-STAT pathway, the principal signaling mechanism for a wide array of cytokines and growth factors (Figure 1). JAK2 mutation activates signaling through MPL (involved in erythrocytosis), EPOR (thrombocytosis), and G-CSFR (neutrophilia) cytokine receptors. Loss of heterozygosity is quite frequent, leading to very high variant allele frequency (VAF) up to $90-100 \%$, particularly in PV.
CALR exon 9 frameshift mutations lead to activated MPL and less markedly G-CSFR, resulting in thrombocytosis but not erythrocytosis. Eighty percent of mutated CALR belong to one of the two following types: a 52 bp deletion, named type 1 , and a 5 bp insertion, named type 2 mutation. The remaining $20 \%$ of cases are classified as type-1, respectively type 2- like mutations, depending on their conformation.

MPL mutations lead to dimerization of the MPL receptor, which in turn causes a constitutional activation of this receptor, with similar results as $J A K 2 \mathrm{~V} 617 \mathrm{~F}$.

In general, $J A K 2$ and $M P L$ mutations have the least positive effect for the patients, followed by CALR type 1-like mutations, whereas the best outcome is observed for CALR type 2-like mutations.

Routinely, molecular investigations are done testing sequentially, beginning with the most common MPN mutations according to their frequency: JAK2V617F, CALR exon 9, and $M P L$, followed by the analysis of $J A K 2$ exon 12 for PV. The frequency of the common driver mutations and variant allele frequencies can help to distinguish PV, ET, and PMF (Table 3). Patients who carry none of these three mutations are classified as "triple-negative" patients, for whom a more in-depth screening is needed for diagnosis, prognosis, and therapy decisions.

\section{Extended panels contributing to risk stratification}

Risk stratification is a key tool for decision-making in any disease with markedly heterogeneous outcomes. In this regard, investigations of additional mutations have contributed enormously in helping to identify high-risk patients. Patients with severe forms of the disease may be cured by allogeneic hematopoietic stem cell transplantation (HSCT), it would therefore be mandatory to identify them early enough not to lose timely this unique therapeutical option. In PMF, this is the case, risk stratification needs a workable scoring system that is as precise as possible but remaining reasonably simple to handle.

For PMF, international collaborations have introduced prognostic scoring systems since 2009, which help decisionmaking regarding treatments like HSCT for high-risk patients. The first system was based on clinical variables. ${ }^{76}$ Subsequently, MIPSS70 and MIPSS70-plus added genetic variables. ${ }^{94}$ In recent years, several scoring systems with diverse molecular and clinical features were published. In 2018, a new tool was developed, ${ }^{95}$ which relies solely on cytogenetic and molecular markers: the genetically inspired prognostic system for primary myelofibrosis (GIPSS). The preferred two-step approach of the authors is that the GIPSS is scored first, stratifying the patients into four prognostic groups (low, intermediate int-1, int-2, high risk) (Table 4). Patients falling into the two intermediate categories are further evaluated using the MIPSS70+ scoring system (Table 5), which allows improved discrimination. This approach represents an easy-to-use system for the first evaluation, with the possibility of refining the findings in a second step. 
Table 3. Driver mutations for BCR-ABL1 negative MPNs. ET, essential thrombocythemia; MPN, myeloproliferative neoplasms; PMF, primary myelofibrosis; PV, polycythemia vera.

\begin{tabular}{|c|c|c|c|c|}
\hline Disease & Mutation & VAF & Frequency & Consequence \\
\hline \multirow[t]{2}{*}{ PV } & JAK2V617F & frequently over $50 \%$ & $95 \%$ & increased erythrocytes, platelets, granulocytes \\
\hline & JAK2 exon 12 & frequently low, 5-15\% & $3 \%$ & increase erythorcytes \\
\hline \multirow[t]{3}{*}{ ET } & JAK2V617F & lower as in PV, about $25 \%$ & $50-60 \%$ & increased erythrocytes, platelets, granulocytes \\
\hline & $\begin{array}{l}\text { CALR exon } 9 \\
\text { CALR exon } 9 \text { type } 1 \\
\text { (or type } 1 \text {-like) } \\
\text { CALR exon } 9 \text { type } 2 \\
\text { (or type } 2 \text {-like) }\end{array}$ & around $40 \%$ & $\begin{array}{l}25 \% \\
55 \% \text { of CALR mut. } \\
45 \% \text { of CALR mut. }\end{array}$ & increased platelets \\
\hline & $\begin{array}{l}\text { MPLW515L/K, other } \\
\text { rare mutations }\end{array}$ & can be low & $3 \%$ & increased platelets \\
\hline \multirow[t]{3}{*}{ PMF } & JAK2V617F & variable, frequently high & $50-60 \%$ & increased erythrocytes, platelets, granulocytes \\
\hline & $\begin{array}{l}\text { CALR exon } 9 \\
\text { CALR exon } 9 \text { type } 1 \\
\text { (or type } 1 \text {-like) } \\
\text { CALR exon } 9 \text { type } 2 \\
\text { (or type } 2 \text {-like) }\end{array}$ & $50(-100 \%)$ & $\begin{array}{l}20 \% \\
75 \% \text { of CALR mut. } \\
13 \% \text { of CALR mut. }\end{array}$ & increased platelets \\
\hline & $\begin{array}{l}\text { MPLW515L/K, other } \\
\text { rare mutations }\end{array}$ & can be low & $3-8 \%$ & increased platelets \\
\hline
\end{tabular}

\section{Precision genomics}

Chronic myelomonocytic leukemia (CMML) provides a good example of the value of precision genomics for prognostication and targeted therapeutic advances based on the implementation of a variety of genetic markers for diagnosis and therapeutic management. ${ }^{96}$ Currently, also in MPNs, progress is made towards personalized predictions of patient's' outcomes and optimal therapeutic decisions. In 2018, eight subgroups of MPNs were identified by comprehensive genomic characterization defining distinct genetic subgroups and by integration with clinical variables. ${ }^{88}$ We can recognize the heterogeneity of MPNs and their prognosis on the combination of the driver and the additional associated mutations. ${ }^{97,98}$ The most common MPN-related mutations affect epigenetic regulation, splicing, and signaling. All these mutations are associated with a poor prognosis and progression of the disease. Many of these genes, such as $D N M T 3 A, A S X L 1$, and TET2, are associated with clonal hematopoiesis of indeterminate potential (CHIP) and therefore are found with increasing incidence in elderly patients, often associated with an increased risk of subsequent hematologic malignancies. ${ }^{99}$ Several studies have shown that the higher the number of adverse mutations is as well for the prognosis significant. ${ }^{97,100}$

The most commonly mutated non-driver genes involved in epigenetic regulation are from two different groups with TET2, DNMT3A, and $I D H 1 / 2$ involved in DNA methylation and $A S X L 1$ and $E Z H 2$, being part of the polycomb repressor complex modulating chromatin structure. In up to $22 \%$ of MPN cases, TET 2 mutations were identified, and patients acquiring the $J A K 2 \mathrm{~V} 617 \mathrm{~F}$ driver mutation prior to the one in TET2 present with a more severe phenotype and are more likely to be diagnosed with PV compared to ET. Furthermore, they suffer from an increased risk of thrombosis. ${ }^{101}$ In less than $10 \%$ of patients, $J A K 2 \mathrm{~V} 617 \mathrm{~F}$ is followed later on by a DNMT3A mutation, more likely associated with PV or MF rather than ET. ${ }^{102}$ Mutations in $I D H 1 / 2, E Z H 2$, and $A S X L 1$ are associated with an increased risk of leukemic transformation. ${ }^{103}$

Table 4. GIPSS: genetically inspired prognostic scoring system. Adapted from Tefferi et al. ${ }^{95}$

\begin{tabular}{|l|l|l|}
\hline Category of features & Feature & score \\
\hline \multirow{2}{*}{ Karyotype } & very high risk (VHR) & 2 \\
\cline { 2 - 3 } & unfavorable & 1 \\
\hline Driver mutation & $\begin{array}{l}\text { absence of CALR type } \\
\text { 1-like mutation }\end{array}$ & 1 \\
\hline High molecular risk (HMR) & ASXL1 mutation & 1 \\
\cline { 2 - 3 } & SRSF2 mutation & 1 \\
\cline { 2 - 3 } & U2AF1Q157 mutation & 1 \\
\hline
\end{tabular}

Mutations in regulators of the splicing machinery generally occur in PMF, mainly affecting U2AF1, SRSF2, ZRSR2, and $S F 3 B 1$ genes. Rarely, cell signaling (SH2B3, CBL, NRAS/ $K R A S$, and PTPN11) or transcription factors, like RUNX1, are affected. The occurrence of these alterations correlates with poor prognosis and disease progression. ${ }^{97,98}$ Mutations in DNA damage response genes, particularly TP53, often associated with complex karyotypes or abnormalities in chromosomes 5 and/or 7, are detected at later stages of the disease and correlate with progression or can be related to previous treatments as demonstrated for PPMID. ${ }^{104}$

Many additional somatic mutations found in MPNs are already present at diagnosis. Therefore, a comprehensive mutation screening at diagnosis provides considerable prognostic information, including the risk of leukemic progression differing significantly among the entities and mutational profiles that supports therapeutic decisions and thereby is a 


\section{healthbook TIMES Oncology Hematology}

key factor for the implementation of precision medicine for the treatment of MPNs.

\section{THERAPEUTIC DECISION MAKING IN MPN PATIENTS}

At the time of initial presentation, MPN patients may have symptoms or complications requiring urgent therapeutic intervention. Generally, at this early time point, peripheral blood values and patient's symptoms are the only information available for therapeutic decisions. Cytoreductive therapy or management of complications such as thromboses, or less commonly, bleeding, may be required. Patients presenting with symptomatic polyglobulia should undergo phlebotomies and/or cytoreduction, likewise, microcirculation problems associated with thrombocytosis require cytoreduction. Hydroxyurea (HU) remains the first-line cytoreductive treatment of choice. ${ }^{105}$ In rare instances, severe life-threatening microcirculation complications due to polyglobulia or thrombocytosis can also be treated by RBC or platelets apheresis, which may be lifesaving in selected cases.$^{106,107}$

Once the MPN diagnostic workup is completed and the diagnosis is established, the specific approach in each individual patient should be considered. Indeed, there is no one way to treat all MPNs, each entity requires specific monitoring and treatment according to the prognosis. Many international guidelines for the therapeutic management of MPNs are available. ${ }^{11,80,108}$ Treatment consists of alleviating systemic symptom burden, controlling disease-proliferation and/or splenomegaly, and prevention of complications while prioritizing patients' quality of life in each decision. Control of general risk factors for thrombosis, including smoking habits, diabetes mellitus, arterial hypertension, and hypercholesterolemia, is in all MPNs recommended.

The prognostication systems specifically designed for each MPN entity can guide the treatment steps. The thrombotic risk has an important weight on therapeutic decisions in patients with PV and ET. On the contrary, in MF, the thrombotic risk is not consistently included in the therapeutic algorithm. In $\mathrm{PV}$, age and history of previous thrombosis are the prognostic factors used to classify patients in low and high-risk categories. Low-risk patients will be mainly managed with phlebotomies and low-dose aspirin, while high-risk will additionally require cytoreduction. ${ }^{11}$ In ET, the IPSET is the recommended prognostic system comprising age, previous thrombosis, cardiovascular risk factors, and $J A K 2 \mathrm{~V} 617 \mathrm{~F}$ mutation (Table 2). Low-risk patients are observed or treated with low doses of aspirin, while high-risk patients will additionally require cytoreduction. ${ }^{76}$

In MF, there are several models to identify prognosis for treatment decisions. Older age, the presence of anemia, leukocytosis, thrombocytopenia, circulating blasts, constitutional symptoms, need for transfusions and bone marrow fibrosis are factors associated with poor prognosis. Furthermore, cytogenetics features, the type of driver mutations, and high-risk mutations contribute in different scoring systems (Table 4 and Table 5). ${ }^{95}$ When molecular information is limited, it will be necessary to apply scores that consider only clinical parameters. ${ }^{109,110}$ In MF, the treatment primarily aims at controlling symptoms, reducing splenomegaly, and fibrosis. Transplant eligible patients must be identified timely; frequently, anti-JAK 2 compounds will be used before transplantation.

Table 5. MIPSS70: mutation-enhanced international prognostic scoring system for transplant-age patients. Adapted from Guglielmelli P et al. ${ }^{94}$

\begin{tabular}{|l|l|l|l|}
\hline Risk category & Total score & $\mathbf{5}$-year OS & Median OS \\
\hline Low-risk & 0 & $94 \%$ & 26.4 years \\
\hline Int-1 & 1 & $73 \%$ & 10.3 years \\
\hline Int-2 & 2 & $40 \%$ & 4.6 years \\
\hline High-risk & 3 & $14 \%$ & 2.6 years \\
\hline
\end{tabular}

In general, in MPNs when cytoreduction is indicated, HU, anagrelide, interferons, ruxolitinib, and fedratinib are options for everyday use with different indications (80). A number of ongoing studies investigating different drugs in the field of MPNs anticipate an exciting future landscape in the management of these diseases. ${ }^{11}$

The treatment of MDS/AML transformed from MPN is difficult; prognosis is in general adverse. ${ }^{112}$ The commonly advanced age of the MPN population and their comorbidities frequently represent treatment limitations. Chemotherapybased schemata for AML represent the standard therapy in fit patients; however, many patients will just receive palliative treatments. ${ }^{113}$ Advances in targeted therapies for AML represent an attractive choice in a field with otherwise few options. HSCT is considered the only curative potential treatment in patients younger than 65 years or as well for older patients still in good general condition. ${ }^{114,115}$ 


\section{CONCLUSION}

Although classic BCR-ABL1 negative myeloproliferative syndromes are a group of diseases with some common aspects, it is extremely important to establish the precise diagnosis of the different entities that compose this group since PV, ET, and MF have different risks, prognosis and require different therapeutical management. Undoubtedly, in recent years, there has been an enormous contribution in the understanding of the molecular aspects of these diseases; likewise, the update of the WHO was an important step in their diagnostic precision. A deep understanding of the laboratory, clinical and complication profile of each entity are valuable contributors for greater accuracy in patient management.

\section{TAKE-HOME MESSAGES}

- In MPN negative for BCR-ABL1, consideration of individual variability is imperative in both diagnosis and treatment of the disease.

- The combination of clinical, morphological, and molecular genetic characteristics is the most appropriate attempt to define MPN.

- Bone marrow morphology remains the central hallmark in the 2016 WHO MPN classification.

- Prognostic systems designed specifically for each MPN entity will guide the steps of treatment.

\section{AUTHOR CONTRIBUTIONS:}

Linet Njue (LN), Naomi Porret (NP), Vera Ulrike Bacher (VUB), Anne Angelillo-Scherrer (AAS) and Alicia Rovó (AR) LN, NP, AAS, VUB and AR wrote the manuscript. LN, NP and AR created the table and figures. All authors revised the manuscript critically and approved the final version.

\section{CONFLICT OF INTEREST:}

$\mathrm{LN}$ : none

NP: none

VUB: none

AAS: Consultancy Vifor Pharmaceuticals

AR: Novartis: Consultancy, Honoraria, Advisory Board, Research Funding; CSL Behring: Research Funding; Alexion: Research Funding; Orphaswiss: Advisory Board. Celgene: Advisory Board Consultancy. AstraZeneca: advisory Board.

1. Blum HE. Individualized medicine 2010. J Cell Mol. Med. 2010; 14(9): 2257-63.

2. Jackson SE et al. Personalised cancer medicine: Personalised Cancer Medicine. Int J Cancer. 2015; 137(2): 262-6.

3. Kiladjian J-J et al. Myeloproliferative neoplasms and personalized medicine: the perfect match? Haematologica. 2015; 100(12): 1493-

4. Michiels JJ et al. Proposal for revised diagnostic criteria of essential thrombocythemia and polycythemia vera by the Thrombocythemia Vera Study Group. Semin Thromb Hemost. 1997; 23(4): 339-47.

5. Yonemitsu $\mathrm{H}$ et al. [Clinical significance of red cell distribution width in polycythemia vera]. Rinsho Byori. 1989; 37(7): 813-8.

6. Ginzburg YZ et al. Dysregulated iron metabolism in polycythemia vera: etiology and consequences. Leukemia. 2018;32(10): 2105-16.

7. Sandes AF et al. Frequency of polycythemia in individuals with normal complete blood cell counts according to the new 2016 WHO classification of myeloid neoplasms. Int J Lab Hematol. 2017; 39(5): 528-31

8. Arber DA et al. The 2016 revision to the World Health Organization classification of myeloid neoplasms and acute leukemia. Blood. 2016;127(20):2391-2405. Blood. 2016; 128(3): 462-3.

9. Geyer HL et al. Distinct clustering of symptomatic burden among myeloproliferative neoplasm patients: retrospective assessment in 1470 patients. Blood. 2014; 123(24): 3803-10.

10. Emanuel RM et al. Myeloproliferative Neoplasm 10. Emanuel RM et al. Myeloproliferative Neoplasm (MPN) Symptom Assessment Form Total Symptom Score: Prospective International Assessment of an Abbreviated
Symptom Burden Scoring System Among Patients With Symptom Burden Scoring System Among Pa
MPNs. J Clin Oncol 2012; 30(33): 4098-103. MPNs. J Clin Oncol 2012; 30(33): 4098-103.
11. McMullin MF et al. A guideline for the diagnosis and 11. McMullin MF et al. A guideline for the diagnosis and
management of polycythaemia vera. A British Society for Haematology Guideline. Br J Haematol. 2019; 184(2): 176-91.

12. Scherber RM et al. Comprehensively understanding fatigue in patients with myeloproliferative neoplasms. Cancer. 2016; 122(3): 477-85.

13. Geyer $H$ et al. Symptomatic Profiles of Patients
Cancer.
With Polycythemia Vera: Implications of Inadequately Controlled Disease. J Clin Oncol. 2016; 34(2): 151-9.

14. Visani $G$ et al. Myelofibrosis with myeloid metaplasia: clinical and haematological parameters predicting survival in a series of 133 patients. BrJ Haematol. 1990; 75 (1):4-9. 15. Cervantes $F$ et al. New prognostic scoring system for 15. Cervantes $F$ et al. New prognostic scoring system for
primary myelofibrosis based on a study of the International primary myelofibrosis based on a study of the International Working Group for Myelofibrosis

\section{Blood. 2009; 113(13): 2895-901.}

16. Patel $\mathrm{AB}$ et al. JAK2 ex13InDel drives oncogenic transformation and is associated with chronic eosinophilic leukemia and polycythemia vera. Blood. 2019; 134(26): 2388-98.

17. Thiele J et al. Diagnostic impact of bone marrow histopathology in polycythemia vera (PV). Histol Histopathol. 2005; 20(1):317-28.

18. Lamy $T$ et al. Inapparent polycythemia vera: an unrecognized diagnosis. Am J Med. 1997; 102(1): 14-20. unrecognized diagnosis. Am J Med. $1997 ; 102(1): 14-20$.
19. Barbui $T$ et al. Masked polycythemia vera (mPV): results of an international study. Am J Hematol. 2014; 89(1): 52-4.

20. Alvarez-Larran A et al. Red cell mass measurement in patients with clinically suspected diagnosis of polycythemia vera or essential thrombocythemia. Haematologica. 2012; 97(11): 1704-7.

21. Cassinat B et al. Classification of myeloproliferative disorders in the JAK2 era: is there a role for red cell mass? Leukemia. 2008; 22(2): 452-3.

22. Maslah N et al. Masked polycythemia vera: analysis of a single center cohort of 2480 red cell masses. Haematologica. 2020, 105(3): e95-7.

23. Valla D et al. Etiology of portal vein thrombosis in adults. A prospective evaluation of primary myeloproliferative disorders. Gastroenterology. 1988; 94(4): 1063-9.

24. Hussein $\mathrm{K}$ et al. JAK2(V617F) allele burden discriminates essential thrombocythemia from a subset of prefibrotic-stage primary myelofibrosis. Exp Hematol. 2009; 37(10): 1186-1193.e7.

25. Barbui $T$ et al. Survival and disease progression in essential thrombocythemia are significantly influenced by accurate morphologic diagnosis: an international study. J Clin Oncol. 2011;29(23): 3179-84.

26. Barbui $T$ et al. Disease characteristics and clinical outcome in young adults with essential thrombocythemia versus early/prefibrotic primary myelofibrosis. Blood. 2012; 120(3): 569-71.

27. Guglielmelli $P$ et al. Presentation and outcome of patients with 2016 WHO diagnosis of prefibrotic and overt primary myelofibrosis. Blood. 2017; 129(24): 3227-36.

28. Hehlmann Ret al. Essential thrombocythemia. Clinical characteristics and course of 61 cases. Cancer. 1988. 61(12): 2487-96

29. Carobbio A et al. Blood tests may predict early primary myelofibrosis in patients presenting with essential thrombocythemia. Am J Hematol. 2012; 87(2): 203-4.

30. Pereira $\mathrm{A}$ et al. [Primary myelofibrosis: description of a series of 53 patients]. Med Clin. (Barc.). 1989; 92(14): $521-5$.

31. Mazzotta $S$ et al. Increased serum lactate dehydrogenase isoenzymes in Ph-negative chronic myeloproliferative diseases: a metabolic adaptation? Hematology. 2006, 11(4): $239-44$ 32. Xin C-H et al. Analysis on 71 patients with
polycythemia vera. Zhongguo Shi Yan Xue Ye Xue Za Zhi. 2012; 20(3): 667-70.

33. Cherry $M$ et al. Patients with polycythemia vera and essential thrombocythemia with prior malignancy do not have significantly worse outcome. Leuk Res. 2013; 37(11): 1472-6.

34. Shah $S$ et al. Marked elevation of serum lactate dehydrogenase in primary myelofibrosis: clinical and prognostic correlates. Blood Cancer J. 2017; 7(12): 657.

35. Lussana $F$ et al. Inflammation and myeloproliferative neoplasms. J Autoimmun. 2017; 85: 58-63.

neoplasms. Jutoimmun. 2017; 85: 58-63. thrombocythemia and polycythemia vera: different role of $\mathrm{C}$-reactive protein and pentraxin 3. Haematologica. 2011 96(2):315-8.

37. Lussana $\mathrm{F}$ et al. Driver mutations (JAK2V617F MPLW515L/K or CALR), pentraxin-3 and C-reactive protein in essential thrombocythemia and polycythemia vera. J Hematol Oncol. 2017; 10(1): 54

38. Economopoulos $\mathrm{T}$ et al. Monoclonal gammopathy in chronic myeloproliferative disorders. Blut. 1989; $58(1)$ 7-9.

39. Randi ML et al. Polycythemia vera and essential 


\section{healthbook TIMES Oncology Hematology}

thrombocythemia with monoclonal gammopathy:
experience of a single institution. Haematologica. 2001; 86(7): 769-70.

40. Javorniczky NR et al. Prevalence and characteristics of myeloproliferative neoplasms with concomitant monoclonal gammopathy. Leuk Res. 2020; 98: 106454.

41. Le Clech L et al. The presence of monoclonal 41. Le Clech $\mathrm{L}$ et al. The presence of monoclonal
gammopathy in Ph-negative myeloproliferative neoplasms gammopathy in Ph-negative myeloproliferative neoplasms
is associated with a detrimental effect on outcomes. Leuk is associated with a detrimental effect
Lymphoma. 2017; 58(11): 2582-7.

Lymphoma. 2017; 58(11): $2582-7$.
42. Pizzi M et al. Recombinant interferon- $\alpha$ in myelofibrosis reduces bone marrow fibrosis, improves its morphology and is associated with clinical response. Mod Pathol. 2015; 28(10): 1315-23.

43. Martin K. Risk Factors for and Management of MPNAssociated Bleeding and Thrombosis. Curr Hematol Malig Rep. 2017; 12(5): 389-96.

44. Kc D et al. The underappreciated risk of thrombosis and bleeding in patients with myelofibrosis: a review. Ann Hematol. 2017; 96(10): 1595-604

45. Abdulkarim $\mathrm{K}$ et al. Risk factors for vascula complications and treatment patterns at diagnosis of 238 PV and ET patients: Real-world data from the Swedi MPN Registry. Eur J Haematol. 2017; 98(6): 577-83. 46. Angona A et al. [Essential thrombocythemia: baseline characteristics and risk factors for survival and thrombosis in a series of 214 patients]. Med Clin (Barc). 2015; 144(6): $247-53$.

47. Bertozzi I et al. Thromboses and hemorrhages are common in MPN patients with high JAK2V617F allele burden. Ann Hematol. 2017; 96(8): 1297-302.

48. Cerquozzi $S$ et al. Risk factors for arterial versus venous thrombosis in polycythemia vera: a single center experience in 587 patients. Blood Cancer J. 2017; 7(12): 662.

49. Kaifie A et al. Bleeding, thrombosis, an anticoagulation in myeloproliferative neoplasms (MPN) analysis from the German SAL-MPN-registry. J Hematol Oncol. 2016; 9: 18

50. Rungjirajittranon $\mathrm{T}$ et al. A systematic review and meta-analysis of the prevalence of thrombosis and bleeding at diagnosis of Philadelphia-negative myeloproliferative neoplasms. BMC Cancer. 2019; 19(1): 184.

51. Kander EM et al. Bleeding complications in BCR-ABL negative myeloproliferative neoplasms: prevalence, type, and risk factors in a single-center cohort. Int J Hematol. 2015; 102(5): 587-93.

52. Stein BL et al. From Budd-Chiari syndrome to acquired von Willebrand syndrome: thrombosis and bleeding complications in the myeloproliferative neoplasms. Blood. 2019; 134(22): 1902-11.

53. Rottenstreich $A$ et al. Factors related to the developmen of acquired von Willebrand syndrome in patients with essential thrombocythemia and polycythemia vera. Eur J Intern Med. 2017; 41: 49-54.

54. Finazzi G et al. Incidence and risk factors for bleeding in 1104 patients with essential thrombocythemia or prefibrotic myelofibrosis diagnosed according to the 2008 prefibrotic myelofibrosis diagnosed according

55. Hernández-Boluda J-C et al. Oral anticoagulation to prevent thrombosis recurrence in polycythemia vera and essential thrombocythemia. Ann Hematol. 2015; $94(6)$ 911-8

56. De Stefano V et al. High rate of recurrent venous thromboembolism in patients with myeloproliferative neoplasms and effect of prophylaxis with vitamin $\mathrm{K}$ antagonists. Leukemia. 2016; 30(10): 2032-8.

57. Landolfi R et al. Efficacy and safety of low-dose aspirin in polycythemia vera. N Engl J Med. 2004; 350(2): 11424.

58. Gisslinger $\mathrm{H}$ et al. Anagrelide compared with hydroxyurea in WHO-classified essential thrombocythemia: the ANAHYDRET Study, a
randomized controlled trial. Blood. 2013;121(10): 1720randi 8

59. Tefferi A et al. Long-term survival and blast transformation in molecularly annotated essential thrombocythemia, polycythemia vera, and myelofibrosis. Blood. 2014; 124(16): 2507-13; quiz 2615.

60. Setiadi $\mathrm{H}$ et al. Endothelial signaling by neutrophilreleased oncostatin $\mathrm{M}$ enhances P-selectin-dependent inflammation and thrombosis. Blood Adv. 2019; 3(2): 168-83.

61. Marchioli $\mathrm{R}$ et al. Cardiovascular events and intensity of treatment in polycythemia vera. N Engl J Med. 2013; 368(1): 22-33.

62. Barbui $\mathrm{T}$ et al. White blood cell counts and thrombosis in polycythemia vera: a subanalysis of the CYTO-PV study Blood. 2015; 126(4): 560-1.

63. Campbell PJ et al. Correlation of blood counts with vascular complications in essential thrombocythemia: analysis of the prospective PT1 cohort. Blood. 2012 120(7): 1409-11.

64. Carobbio A et al. Leukocytosis and thrombosis in essential thrombocythemia and polycythemia vera: a
systematic review and meta-analysis. Blood Adv. 2019;
3(11): 1729-37.

65. Barbui $\mathrm{T}$ et al. Thrombosis in primary myelofibrosis: incidence and risk factors. Blood. 2010; 115(4): 778-82.

66. Landolfi $\mathrm{R}$ et al. Increased thromboxane biosynthesis in patients with polycythemia vera: evidence for aspirinsuppressible platelet activation in vivo. Blood. 1992; 80(8): 1965-71.

67. Zhang Y et al. Thrombosis among 1537 patients with JAK2V617F -mutated myeloproliferative neoplasms: Risk factors and development of a predictive model. Cancer Med. 2020; 9(6): 2096-105.

68. Falanga A et al. Thrombotic disease in the myeloproliferative neoplasms. Hematol Am Soc Hemato Educ Program. 2012; 2012: 571-81.

69. Dentali $F$ et al. Cerebral venous thrombosis and myeloproliferative neoplasms: results from two large databases. Thromb. Res. 2014; 134(1): 41-3.

70. Akpan IJ et al. Splanchnic Vein Thrombosis in the Myeloproliferative Neoplasms. Curr Hematol Malig Rep. 2018; 13(3): 183-90

71. Smalberg JH et al. Myeloproliferative neoplasms in Budd-Chiari syndrome and portal vein thrombosis: a metaanalysis. Blood. 2012; 120(25): 4921-8.

72. Stein BL et al. Age-related differences in disease characteristics and clinical outcomes in polycythemia vera. Leuk Lymphoma. 2013; 54(9): 1989-95.

73. How J et al. Distinct clinical, laboratory and molecular features of myeloproliferative neoplasm patients with splanchnic vein thrombosis. Br J Haematol. 2018; 183(2) 310-3.

74. Poisson J et al. Selective testing for calreticulin gene mutations in patients with splanchnic vein thrombosis: A prospective cohort study. J Hepatol. 2017; 67(3): 501-7.

75. Rumi E et al. Diagnosis, risk stratification, and response evaluation in classical myeloproliferative neoplasms. Blood. 2017; 129(6): 680-92

76. Barbui T et al. Development and validation of an International Prognostic Score of thrombosis in World Health Organization-essential thrombocythemia (IPSETthrombosis). Blood. 2012; 120(26): 5128-33.

77. Barbui $T$ et al. Practice-relevant revision of IPSET thrombosis based on 1019 patients with WHO-defined essential thrombocythemia. Blood Cancer J. 2015; 5: e369.

78. Haider $\mathrm{M}$ et al. Validation of the revised International Prognostic Score of Thrombosis for Essential Prognostic Score of Thrombosis for Essential
Thrombocythemia (IPSET-thrombosis) in 585 Mayo Thrombocythemia (IPSET-thrombosis) in 585
Clinic patients. Am J Hematol. 2016; 91(4):390-4.

Clinic patients. Am J Hematol. 2016; 91 (4): 390-4.
79. Finazzi G et al. Calreticulin mutation does not modify the IPSET score for predicting the risk of thrombosi among 1150 patients with essential thrombocythemia. Blood. 2014; 124(16): 2611-2.

80. Barbui $\mathrm{T}$ et al. Philadelphia chromosome-negative classical myeloproliferative neoplasms: revised management recommendations from European LeukemiaNet. Leukemia. 2018; 32(5): 1057-69.

81. Noor SJ et al. Myeloid blastic transformation of myeloproliferative neoplasms--a review of 112 cases. Leuk Res. 2011;35(5): 608-13.

82. Kreipe HH. [Precursors of acute leukemia: myelodysplastic syndromes and myeloproliferative neoplasms]. Pathol. 2011;32 Suppl 2: 271-6.

83. Mesa RA et al. Primary myelofibrosis (PMF), pos polycythemia vera myelofibrosis (post-PV MF), post essential thrombocythemia myelofibrosis (post-ET MF) blast phase PMF (PMF-BP): Consensus on terminology by the international working group for myelofibrosis research and treatment (IWG-MRT). Leuk Res. 2007; 31(6) 737-40.

84. Szuber N et al. 3023 Mayo Clinic Patients With Myeloproliferative Neoplasms: Risk-Stratified Comparison of Survival and Outcomes Data Among Disease Subgroups. Mayo Clin Proc. 2019. 94(4):599-610.

85. Björkholm $M$ et al. Treatment-related risk factor for transformation to acute myeloid leukemia and myelodysplastic syndromes in myeloproliferativ neoplasms. J Clin Oncol. 2011; 29(17): 2410-5. 86. Tefferi A et al. Targeted deep sequencing in
polycythemia vera and essential thrombocythemia. Blood Adv. 2016; 1(1): 21-30.

87. Al Assaf C et al. Analysis of phenotype and outcome in essential thrombocythemia with CALR or JAK2 mutations. Haematologica. 2015; 100(7): 893-7.

88. Grinfeld $J$ et al. Classification and Personalized Prognosis in Myeloproliferative Neoplasms. N Engl J Med. 15): $1416-30$

89. Nowell PC et al. Chromosome studies in human leukemia. II. Chronic granulocytic leukemia.J Natl Cancer Inst. 1961; 27: 1013-35.

90. James $C$ et al. A unique clonal JAK2 mutation leading to constitutive signalling causes polycythaemia vera. Nature. 2005; 434(7037): 1144-8.

91. Baxter EJ et al. Acquired mutation of the tyrosine kinase JAK2 in human myeloproliferative disorders. Lancet. 2005 365(9464): 1054-61.

92. Pikman $\mathrm{Y}$ et al. MPLW515L is a novel somatic activating mutation in myelofibrosis with myeloid metaplasia. PLoS Med. 2006; 3(7): e270

93. Nangalia J et al. Somatic CALR mutations in myeloproliferative neoplasms with nonmutated JAK2. N Engl J Med. 2013; 369 (25): 2391-405.

94. Guglielmelli P et al. MIPSS70: Mutation-Enhanced International Prognostic Score System for TransplantationAge Patients With Primary Myelofibrosis. J Clin Oncol. $2018 ; 36(4): 310-8$

95. Tefferi A et al. GIPSS: genetically inspired prognostic scoring system for primary myelofibrosis. Leukemia. 2018 32(7): 1631-42.

96. Mangaonkar AA et al. Advances in chronic myelomonocytic leukemia and future prospects: Lessons learned from precision genomics. Adv Cell Gene Ther 2019; 2(2)

97. Vainchenker W et al. Genetic basis and molecular pathophysiology of classical myeloproliferative neoplasms. Blood. 2017: 129(6): 667-79.

98. Kjær L. Clonal Hematopoiesis and Mutations of Myeloproliferative Neoplasms. Cancers. 2020; 12(8).

99. Bowman RL et al. Clonal Hematopoiesis and Evolution to Hematopoietic Malignancies. Cell Stem Cell. 2018 22(2): $157-70$

100. Tefferi A et al. Genetic Risk Assessment in Myeloproliferative Neoplasms. Mayo Clin Proc. 2017, 92(8): 1283-90.

101. Ortmann CA et al. Effect of mutation order on myeloproliferative neoplasms. N Engl J Med. 2015; 372(7): 601-12.

102. Nangalia J et al. DNMT3A mutations occur early or late in patients with myeloproliferative neoplasms and mutation order influences phenotype. Haematologica. 2015; 100(11): e438-442.

103. Lasho TL et al. Targeted next-generation sequencing in blast phase myeloproliferative neoplasms. Blood Adv. $2018 ; 2(4): 370-80$

104. Higgins A et al. Genetic and Genomic Landscape of Secondary and Therapy-Related Acute Myeloid Leukemia. Genes. 2020; 11(7).

105. Sankar K et al. Do All Patients With Polycythemia Vera or Essential Thrombocythemia Need Cytoreduction? J Natl Compr Cancer Netw. 2018; 16(12): 1539-45.

106. Parra Salinas I et al. Therapeutic erythroapheresis: Experience in patients with polycythemia vera and secondary erythrocytosis. Med Clin (Barc). 2020; 154(1): $16-9$

107. Boddu P et al. The role of thrombocytapheresis in the contemporary management of hyperthrombocytosis in myeloproliferative neoplasms: A case-based review. Leuk Res. 2017; 58: 14-22.

108. Mesa RA et al. NCCN Guidelines Insights: Myeloproliferative Neoplasms, Version 2.2018. J Nat Compr Cancer Netw. 2017; 15(10): 1193-207.

109. Passamonti $F$ et al. A dynamic prognostic model to predict survival in primary myelofibrosis: a study by the IWG-MRT (International Working Group for Myeloproliferative Neoplasms Research and Treatment) Blood. 2010; 115(9): 1703-8.

110. Gangat $N$ et al. DIPSS plus: a refined Dynamic International Prognostic Scoring System for primary myelofibrosis that incorporates prognostic information from karyotype, platelet count, and transfusion status. J. Clin. Oncol. 2011; 29(4): 392-7.

111. Vannucchi $A M$ et al. Emerging treatments for classica myeloproliferative neoplasms. Blood. 2017; 129(6): 693703.

112. Kennedy JA et al. Treatment outcomes following in Philadelphia-negative myeloproliferative neoplasms. Blood. 2013; 121(14): 2725-33.

113. Andriani A et al. Treatment of Philadelphia-negative myeloproliferative neoplasms in accelerated/blastic phase with azacytidine. Clinical results and identification of prognostic factors. Hematol Oncol. 2019; 37(3): 291-5. 114. Kröger $\mathrm{NM}$ et al. Indication and management of allogeneic stem cell transplantation in primary myelofibrosis: a consensus process by an EBMT/ELN internatio

115. Daghia $G$ et al. Allogeneic stem cell transplantation for myelofibrosis patients aged $\geq 65$ years. Eur J Haematol. 2019; 103(4): 370-8.

116. Jia $R$ et al. Progress in elucidation of molecular pathophysiology of myeloproliferative neoplasms and its application to therapeutic decisions. Int J Hematol. 2020 111(2): 182-91. 